Ergod. Th. \& Dynam. Sys. (1992), 13, 45-64

Printed in Great Britain

Copyright (C) 1993 Cambridge University Press

\title{
Integer Cantor sets and an order-two ergodic theorem
}

\author{
ALBERT M. FISHER \\ University of Göttingen, Lotzestr. 13, D3400 Göttingen, Germany
}

(Received 1 December 1989 and revised 1 December 1991)

Abstract. Let $M \subseteq \prod_{-\infty}^{\infty}\{0,1\}$ denote the orbit closure, under the left shift $\sigma$, of the sequence ... (all zeroes) ...101000101000000000101 ... corresponding to the integer Cantor set $[C]=\left\{\sum_{i=0}^{N} a_{i} 3^{i}: a_{i}=0\right.$ or $\left.2, N \in \mathbb{N}\right\}$. We prove that with respect to the infinite invariant measure $\rho$, which is the unique normalized non-atomic invariant measure on $M$, for every $f \in L^{1}(M, \rho)$, for $\rho$-a.e. $x \in M$,

$$
\lim _{N \rightarrow \infty} \frac{1}{\log N} \sum_{k=1}^{N} \frac{S_{k}(f(x))}{k^{d}} \frac{1}{k}=c \int_{M} f d \rho,
$$

where $d=\log 2 / \log 3$, and $c$ is the almost-sure value of the right-hand order-two density of the middle-third Cantor set. The proof uses renormalization to a scaling flow, plus identification of $(M, \sigma)$ as a tower over the Kakutani-von Neumann dyadic odometer.

\section{Introduction}

In this paper we prove an almost-sure ergodic theorem for a certain infinite measurepreserving transformation. The proof uses the Hopf ratio ergodic theorem plus a renormalization argument, based on the fractal-like structure of the return times to a set of finite measure. This idea has its roots in [Be-Fi] and the notion introduced there of order-two density for Cantor sets.

Related theorems have thus far been proved for several examples: some random walk and Markov-like examples, and also Boole's transformation [Aa-De-Fi], and for the horocycle flow for a Fuchsian group of second type [Fi 3]. The example of the present paper is closely connected to that of the horocycle flow (as explained in the last section); the other examples are handled by quite different methods.

Infinite measure transformations exhibit much more varied behavior than the finite case, and so one should not expect a single general theorem of this type to hold. Instead it seems one needs to closely study specific examples, and then perhaps move toward a more general theory. Basic references on the variety of behavior are Aaronson's papers [Aa 1, Aa 2, Aa 3]; see the remarks in $\$ 2$.

For all the examples mentioned previously the theorem has this form: first one normalizes by the 'dimension' of return times, and then one applies a strong averaging method, in this case the Hardy-Riesz log average. Since this is an order-two averaging method in the sense of [Fi 1,2], we call the theorem an order-two ergodic theorem. 
Here is our example. Let $a=\ldots a_{-1} . a_{0} a \ldots$ denote the sequence $\ldots 0000.101000101 \ldots$ in $\Sigma \equiv \prod_{-\infty}^{\infty}\{0,1\}$, associated with the integer Cantor set $[C] \equiv\left\{\sum_{i=0}^{N} c_{i} 3^{i}: N \in \mathbb{N}, c_{i}=0\right.$ or 2$\}$. Now let $M$ be the orbit closure in $\Sigma$ of the sequence $a$, under the left shift $\sigma$. We will show that there is a unique non-atomic ergodic invariant measure $\rho$ on $M$, up to multiplication by a constant; we normalize by setting $\rho([1])=1$ where [1] $=\left\{b \in M: b_{0}=1\right\}$, and note that $\rho(M)=\infty$.

Now we describe the theorem. Let $d=\log 2 / \log 3$, which is the (integer) dimension of $[\mathbf{C}]$, defined to be

$$
\lim _{n \rightarrow \infty} \log \#([C] \cap[0, n]) / \log n
$$

and let $c$ be the almost-sure value for the one-sided order-two density of the middle-third Cantor set $C \subseteq[0,1]$. See [Be-Fi] for an introduction to the properties of integer dimension and for the proof of the existence of $c$.

We will prove the following.

THEOREM. (An order-two ergodic theorem.) For every $f \in L^{1}(M, \rho)$, we have for a.e. $x \in M$,

$$
\lim _{N \rightarrow \infty} \frac{1}{\log N} \sum_{k=1}^{N} \frac{S_{k}(f(x))}{k^{d}} \frac{1}{k}=c \int_{M} f(x) d \rho(x) .
$$

Here $S_{k}$ denotes the partial sum, $S_{k}(f(x)) \equiv \sum_{i=0}^{k-1} f\left(\sigma^{i} x\right)$. The log average is consistent with the Cesáro average but stronger, and is a natural choice for averaging any object with some self-similarity, since it is the exponential conjugate of the Cesáro average; see [Fi 1,2] for background.

Acknowledgments. We wish to thank Jon Aaronson, Tim Bedford, Mariusz Urbański, Michel Dekking and Manfred Denker for discussions, and Dan Rudolph for supplying a key observation: that the base of the tower could be coded as the Kakutani-von Neumann odometer. This allows one to prove the type of unique ergodicity mentioned above, and also leads to the horocycle example, which is briefly described at the end of the last section and will be treated more fully elsewhere.

2. The ratio ergodic theorem, dimension and order-n density

In this section we develop some basic material which is also needed in [Aa-De-Fi, Fi 3]. First we recall Hopf's ratio ergodic theorem, then use it to prove properties for dimension and order- $n$ density.

2.1. Ratio theorem. We will use the abbreviation cempt for a conservative, ergodic measure-preserving transformation $T$, of an infinite measure space $(M, \mathscr{A}, \rho)$ which is non-atomic and $\sigma$-finite.

Hopf's ratio ergodic theorem ([Ho], ch. 4) states, that for a cempt one has the following.

TheOREM 1. (Hopf.) Let $f, g \in L^{1}(M, \rho)$ with $\int_{M} g d \rho \neq 0$. Then for a.e. $x \in M$,

$$
\lim _{n \rightarrow \infty} \frac{\sum_{i=1}^{n} f\left(T^{i} x\right)}{\sum_{i=1}^{n} g\left(T^{i} x\right)}=\frac{\int f}{\int g} .
$$


Note. For the special case of $A, B \in \mathscr{A}$ and $f=\chi_{B}, g=\chi_{A}$ this turns out to be surprisingly easy to prove. Assume $B \subseteq A$ (otherwise replace $A$ by $B \cup A$ ), and build the tower (or Kakutani skyscraper) $M=A \cup(T(A) \backslash A) \cup \cdots$ over $A$. Since $T$ is ergodic, so is the induced map $\left(A, T_{A}, \rho_{A}\right)$ where $\rho_{A}=\rho / \rho(A)$ is the normalized measure. By the Birkhoff ergodic theorem applied to $T_{A}$, the frequency $x \in A$ spends in $B$ is $\rho_{A}(B)=\int f / \int g$. This is also the relative frequency of $B$ to $A$ along the full orbit in the tower. Finally, the conclusion passes immediately from a.e. $x \in A$ over to a.e. $x \in M$.

Remarks. In one sense this is a good analogue in the infinite measure setting for the finite measure ergodic theorem (Birkhoff's theorem), since it is also valid for the finite measure case and moreover there, taking $g \equiv 1$ (if $\rho M=1$ ), it reduces exactly to Birkhoff's theorem. But from a philosophical point of view it is quite different, since it says nothing about the time average behavior of the sequence $f\left(T^{i} x\right)$. One does almost get a time-average statement from the ratio theorem as follows. Taking $g(x)=\chi_{A}(x)$ where $A \subseteq M$ has measure one, and setting $a(x, n) \equiv$ $\sum_{i=1}^{n} g\left(T^{i} x\right)$, the theorem reads: for every $f \in L^{1}$, for a.e. $x$,

$$
\frac{1}{a(n, x)} \sum_{i=1}^{n} f\left(T^{i} x\right) \rightarrow \int_{M} f d \rho .
$$

The problem with this however is that the normalizing sequence $a(n, x)$ is very definitely $x$-dependent. In fact, by a theorem of Aaronson ([Aa 2] Theorem 2), for a cempt there can never exist a universal sequence $a(n)$ which gives a.s. convergence. Nevertheless one can make further progress for some classes of cempts (a notable exception being the 'squashable' transformations of Hajian, Ito and Kakutani [Ha-I-K, Aa 3]; see Proposition 7 below).

One has the following positive results, for certain cempts, given a careful choice of $a(n)$ : distributional limits of $\left(S^{n} f\right) / a(n)$ [Aa 4], upper and lower envelopes [Aa-De 1,2] and the following sort of Cesàro-average version of convergence in measure: $\forall f \in L^{1}, \forall$ subsequence $n_{k}$, there exists a further subsequence $n_{l}$ such that $\left(\left(S^{n_{1}} f\right)(x)\right) / a\left(n_{l}\right)$ converges Cesàro a.s. to $\int_{M} f d \rho[\mathbf{A a}$ 3, Aa-S]. When this latter property holds for some sequence $a(n),(M, \rho, T)$ is called weakly homogeneous, and $a(n)$ is termed the asymptotic type of $T$ (defined up to asymptotic equivalence, i.e. up to ratios converging to 1 ).

To move closer to the time-average spirit of the Birkhoff theorem, our idea is to begin with the sequence $S^{n} f / a(n)$ for an appropriate choice of $a(n)$, and then to smooth out the fluctuations by applying a strong form of averaging method. The Hardy-Riesz log averages fit that role perfectly since they extend in a natural way one's usual intuitive notion of time average, given by the Cesàro average (see the discussion in [Fi 1,2]).

This seems to be an especially suitable method when the normalizing sequence is of the form $a(n)=c n^{d}$, because one then has a strong analogy with Hausdorff dimension and measure, which can in turn suggest a strategy for proving convergence. In this case we choose to normalize by $n^{d}$ rather than $c n^{d}$, for reasons explained at the end of this section. In the present paper we restrict attention to this case; 
some examples which instead fit the closely related situation where $a(n)$ is regularly varying will be studied in [Aa-De-Fi].

2.2. Dimension. We recall from [Be-Fi] that the dimension $\operatorname{dim}(F)$ of $F \subseteq \mathbb{N}$ is $\lim _{n \rightarrow \infty} \log N_{n} / \log n$ (if the limit exists), where $N_{n}=N_{n}(F)=\#(F \cap\{1,2, \ldots, n\})$. (Note that $0 \leq d \leq 1$, since this is true for each $n$ ).

Let $(M, \mathscr{A}, \rho, T)$ be a cempt. Associate with a set $A \in \mathscr{A}$ and a point $x \in M$ the $0-1$ sequence $Y_{k}(A, x) \equiv \chi_{A}\left(T^{k} x\right)$, and the integer set $F(A, x)=\left\{k: Y_{k}=1\right\}$. We will show the following.

Proposition 2. If for some fixed $A \in \mathscr{A}$ with $0<\rho A<\infty$, the dimension of $F(A, x)$ exists for $\rho$-a.e. $x \in A$, then:

(i) it is a.s. constant $(=d)$ on $M$

(ii) for any set $B \in \mathscr{A}$ of finite positive measure, $\operatorname{dim}(F(B, x))=d$ for a.e. $x \in M$.

Proof. The statement $\operatorname{dim}(F)=d$ is equivalent to: for every $\varepsilon>0, \exists n_{0}$ such that for all $n>n_{0}$,

$$
\begin{gathered}
n^{d-\varepsilon}<N_{n}<n^{d+\varepsilon} \quad \text { and therefore to: } \\
\lim _{n \rightarrow \infty} N_{n} / n^{d+\varepsilon}=0 \quad \lim _{n \rightarrow \infty} N_{n} / n^{d-\varepsilon}=+\infty .
\end{gathered}
$$

Now the set of $x$ such that either of these holds for some fixed $d$ is invariant and measurable. Hence by ergodicity the dimension is a.s. constant, proving (i). And by the ratio ergodic theorem, for $n$ large, $N_{n}(x, A)=N_{n}(x, B)(1 \pm \tilde{\varepsilon})$ so dividing by $n^{d+\varepsilon}$ and $n^{d-\varepsilon}$, both go to zero or infinity together, proving (ii).

In this situation we will say the dimension exists for the return sequence of $(M, T, \rho)$.

We will use the terms measure-preserving homomorphism or m.p. factor map to mean a measurable map of infinite mpts which preserves the measure and is a.s. onto. Following Aaronson [Aa 1,5], on the other hand a homomorphism is also allowed to multiply the measure by a constant, and two infinite mpts are similar if there is a third mpt which has each as a factor (or equivalently, if there is a joining of the two transformations). Note: by a theorem of Aaronson and Furstenberg, similarity is an equivalence relation [A a 5].

Proposition 3. If the dimension exists for a cempt $(M, \mathscr{A}, T, \rho)$, then:

(i) it exists for $(M, \mathscr{A}, T, \alpha \cdot \rho)$ where $\alpha$ is any positive real number; and it has the same value

(ii) it exists with the same value for any similar cempt.

Proof. Immediate from Proposition 2, if the joining given by the similarity happens to be ergodic. If not, following the proof of Theorem 2.4 in [Aa 1] (similarity invariance for asymptotic type), one first applies a theorem of Krengel to produce an ergodic decomposition of the joining, each of whose components is a joining of factors of the two maps.

Therefore the existence of dimension, and its value if it exists, are invariants for similarity of transformations. Next we study a finer invariant. 


\subsection{Order-n density}

Theorem 4. Let $(M, \mathscr{A}, \rho, T)$ be a cempt. Assume that there exists $d>0$ such that for some $g \in L^{1}(M, \rho)$ with $\int_{M} g \neq 0$,

$$
\lim _{N \rightarrow \infty} \frac{1}{\log N} \sum_{k=1}^{N} \frac{\left(S_{k}(g(x))\right.}{k^{d}} \frac{1}{k} \quad \text { converges, for a.e. } x \in M \text {. }
$$

Then

(i) this limit is constant a.s., and writing it as $c \cdot \int_{M} g$,

(ii) for every $f \in L^{1}(M, \rho)$, for a.e. $x$, for the same constant $c$,

$$
\lim _{N \rightarrow \infty} \frac{1}{\log N} \sum_{k=1}^{N} \frac{S_{k} f(x)}{k^{d}} \frac{1}{k}=c \int_{M} f d \rho .
$$

Proof. This is nearly the same as for Proposition 2. (i) Constancy follows from ergodicity, since the limit is an invariant measurable function of $x$. (ii) For fixed $x$, given $\varepsilon>0, \exists n_{0}$ such that for all $k>n_{0},\left(S_{k} f\right)(x)=(1 \pm \varepsilon) \cdot\left(\left(S_{k} g\right)(x)\right) \cdot\left(\int f / \int g\right)$. The same holds upon division by $n^{d}$, and convergence for $f$ follows immediately.

We recall from [Fi 2] that the Hardy-Riez log averaging operator is the discretetime version $\left[A_{\varphi}^{2}\right]$ of the order-two averaging operator $A_{\varphi}^{2}$ where $\varphi(x)=e^{-x} \chi_{[0, \infty)}(x)$ and where in general, for $\Psi \geq 0$ with integral one, $\left(A_{\Psi}^{(n)} h\right)(t) \equiv$ $\left(\Psi *\left(h \circ \exp ^{(n)}\right)\right) \circ \log { }^{(n)}(t)$, for $h \in L^{\infty}(\mathbb{R})$. Therefore by Wiener's Tauberian Theorem, and Lemma 2.2 of [Fi 1], once one has convergence for $\boldsymbol{A}_{\varphi}^{(n)}$ it follows automatically for $A_{\Psi}^{(m)}$, for all $m \geq n$. Something similar is true here, although since the function $h(k)=S_{k}(f) / k^{d}$ may be unbounded one must restrict $\Psi$ to be in the Wiener class-and this is the hypothesis needed for Pitt's extension of Wiener's theorem to that case [Wid]. Lemma 2.2 also requires a new proof for unbounded functions; to describe all of this rigorously would take us too far afield at the moment, so we defer those details to a later paper. At any rate, one has the following more general statement:

TheOREm 5. Let $(M, \mathscr{A}, \rho, T)$ be a cempt with $\sigma$-finite infinite measure. Assume there exists $d>0$ such that for some $g \in L^{1}$ with $\int g \neq 0$, for fixed $x$,

$$
\lim _{N \rightarrow \infty}\left(\left[A^{(n)}\right]\left(\frac{S_{k}(g(x))}{k^{d}}\right)\right)(N) \text { converges for a.e. } x \text {, }
$$

then:

(i) this limit is constant a.s., and writing it as $c \cdot \int_{M} g$,

(ii) for every $m \geq n$, for every $\Psi$ in the Wiener class, for every $f \in L^{1}$,

$$
\lim _{N \rightarrow \infty}\left(\left[A_{\Psi}^{(m)}\right]\left(\frac{S_{k}(f(x))}{k^{d}}\right)\right)(N)=c \cdot \int_{M} f .
$$

When this holds, we call it an order-n ergodic theorem.

And as was the case for dimension, one has therefore the following. 
Corollary 6. Let $(M, \mathscr{A}, \rho, T)$ be as above; assume the dimension $d$ exists and that for some $n$, the order-n ergodic theorem holds. Let $c$ be the order-n density of $F(A, x)$ for some $A \in \mathscr{A}$ with measure one. This number is the same for any image (or pre-image) under a measure-preserving homomorphism. For $(M, \mathscr{A}, \alpha \cdot \rho, T)$, the value is $c / \alpha$.

Remarks. Note that in stating Theorem 5 , we could have instead normalized by $a(k)=c k^{d}$ instead of by $k^{d}$. This is the natural thing to do up to homomorphism, since one has then essentially replaced $\rho$ by $1 / c \cdot \rho$, and the constant $c$ then pleasantly turns into 1.

From the geometrical point of view, however, it is probably better not to lose the information given by this constant, and to stay in the category of measure-preserving homomorphisms. As mentioned in [Be-Fi], integer order- $n$ density of dimension $d$ can be thought of as coming from a finitely-additive integer version of Hausdorff $d$-dimensional measure, and so changing $c$ into 1 is equivalent to choosing for each transformation a different normalization for Hausdorff measure (compare [Fa] § 1.4).

Note. The least order, $n$, of averaging operator giving convergence is also a similarity invariant; more generally so is the class of convex changes of scale (see [Fi 1]) whose associated averaging methods converge.

In contrast to the present type of example, for Hajian, Ito and Kakutani's squashable transformations no such time average ergodic theorem will be possible. For a squashable transformation $T$ of $(M, \mathscr{A}, \rho)$ by definition there exists a map $Q: M \rightarrow M$ satisfying two properties: on the one hand $\exists a \neq 1$ such that $\rho \circ Q^{-1}=a \rho$, and on the other hand $T Q=Q T$. The point is (as will be seen in the proof below) that the first property says $Q$ changes the measure, while the second guarantees that $Q$ preserves the return-time structures. Hence no statement of the form 'time average = space average' can hold.

Our proof is based on Aaronson's proof that squashable transformations are not weakly homogeneous (Lemma 2.1 in [Aa 3]).

Proposition 7. For a cempt $T$ which is squashable, for any sequence $a(n)>0$ and any averaging operator $\left[A^{(n)}\right]$ one has either:

(i) for all nonnegative $f \in L^{1}$,

$$
\varlimsup_{N \rightarrow \infty}\left(\left[A^{(n)}\right]\left(\frac{\left(S_{k} f\right)(x)}{a(k)}\right)\right)(N)=\infty \quad \text { a.s. or }
$$

(ii) for all such $f$,

$$
\lim _{N \rightarrow \infty}\left(\left[A^{(n)}\right]\left(\frac{\left(S_{k} f\right)(x)}{a(k)}\right)\right)(N)=0 .
$$

Moreover, the same is true with $A^{n}$ replaced by any averaging operator $A_{\psi}^{c}$ where $c$ is a convex change of scale, or for any of these averages taken along any fixed subsequence $S_{m_{k}}$.

Proof. If the lim sup in (i) is for some $f$ not a.s. $\infty$, then by ergodicity and translation invariance it is a.s. $=c$ for some finite $c \geq 0$. 
Assume for simplicity that $\int f=1$. Then by the Hopf theorem, for any other positive $g \in L^{1}$ the $\lim \sup$ is $c \cdot \int g$. In particular for $A \subseteq M$ with $\rho A=1$, the lim sup for $\chi_{A}$ is $c$. Now on the one hand the lim sup for $\chi_{Q^{-1} A}$ is $c \cdot\left(\mu Q^{-1}(A)\right)=c a \neq c$, but on the other hand since $T Q=Q T$, the sequence of visits of a point $x$ to the set $A$ is identical to the visits of $y \in Q^{-1}\{x\}$ to $Q^{-1} A$, so the time averages must be unchanged, giving a contradiction. The proof is the same for subsequences, and for operators $A_{\psi}^{c}$.

\section{Natural extensions and the scaling flow}

We recall from [Fi 1,2, Be-Fi] the definition of the scaling flow of dimension $d$ : let $\Omega=\left\{g: \mathbb{R}^{+} \rightarrow \mathbb{R}\right.$ continuous with $\left.g(0)=0\right\}$, with topology $\mathscr{T}(\Omega)$ given by uniform convergence on compact sets and Borel $\sigma$-algebra $\mathscr{B}$, and with maps $\tau_{s}: \Omega \rightarrow \Omega$ for $s \in \mathbb{R}$ defined by $\left(\tau_{s} g\right)(t)=g\left(e^{-s} t\right) / e^{-s d}$. We will now define a certain $\tau_{s}$-invariant Borel measure on $\Omega$, which is associated with the geometry of the middle-third Cantor set; the dimension is fixed as $d=\log 2 / \log 3$.

First recall that every measure-preserving transformation of a Lebesgue space has a canonical invertible version, unique up to measure theoretic isomorphism, called its natural extension [Ro]. Let $C \subseteq[0,1]$ be the middle-third Cantor set, $\mu$ the Hausdorff $d$-dimensional measure restricted to $C$, and $S: C \rightarrow C$ the ergodic measurepreserving map defined by $S(x)=3 x(\bmod 1)$. The natural extension of $S$ can be represented as the map $\hat{S}: \hat{C} \rightarrow \hat{C}$ with $\hat{C}=C \times C$, defined by:

$$
\begin{aligned}
S(x, y) & =\left(3 x, \frac{1}{3} y\right) \text { for } x \in\left[0, \frac{1}{3}\right] \\
& =\left(3 x-2, \frac{1}{3} y+\frac{2}{3}\right) \text { for } x \in\left[\frac{2}{3}, 1\right] .
\end{aligned}
$$

Geometrically this is a sort of 'baker's transformation'.

Recall that $S$ is canonically isomorphic to the left shift (with truncation) $\sigma$ on $\Pi^{+} \equiv \Pi_{0}^{\infty}\{0,2\}$, via the map $x \mapsto\left(x_{0}, x_{1}, \ldots\right) \in \Pi^{+}$where $x$ has ternary expansion $x=\sum_{i=0}^{\infty} x_{i} / 3^{i+1}$. With this notation, the map is written $x=\left(. x_{0} x_{1} \ldots\right) \mapsto\left(x_{0}, x_{1}, \ldots\right) \in$ $\Pi^{+}$. The invariant measure for $\hat{S}$ is $\hat{\mu} \equiv \mu \times \mu$, and the corresponding $\sigma$-invariant measure on $\Pi^{+}$is Bernoulli $\left(\frac{1}{2}, \frac{1}{2}\right)$ infinite product measure. The map $\hat{S}: \hat{C} \rightarrow \hat{C}$ corresponds to the full left shift $\sigma$ on $\Pi \equiv \prod_{-\infty}^{\infty}\{0,2\}$, which in turn is the natural extension of $\sigma$ on $\Pi^{+}$. For a point $\hat{x}=(x, y) \in C \times C$, with $x=\left(. x_{0} x_{1} \ldots\right)$ and $y=\left(. y_{0} y_{1} \ldots\right)$, the map from $\hat{C}$ to $\Pi$ is $\hat{x} \mapsto\left(\ldots x_{-2}, x_{-1}, x_{0}, x_{1}, x_{2}, \ldots\right)$ where $\left(x_{-1}, x_{-2}, \ldots\right)=\left(y_{0}, y_{1}, y_{2}, \ldots\right)$. We will also write $\left(\ldots x_{-2} x_{-1}, x_{0} x_{1} \ldots\right)$ for the point $\hat{x}$ in $\hat{C}$.

Now let $Y=\hat{C} \times[0, \log 3]$ with the topological identification $(\hat{x}, \log 3)=(\hat{S}(\hat{x}), 0)$, and let $\Phi_{1}$, denote the special flow (suspension flow) on $Y$; that is, one flows up with unit speed and then jumps back via the map $\hat{S}$ on the Poincaré cross-section $\hat{C} \times\{0\}$. The measure we use is the product of $\hat{\mu}$ with Lebesgue measure; we denote this by $m$. So $\left(Y, \Phi_{t}, m\right)$ is an ergodic flow.

Next we define a continuous bounded function on $Y$. Set

$$
f(\hat{x}, t)=\mu\left[x, x+e^{-t}\right] / e^{-t d} \text { for } t \in[0, \log 3),
$$

where $\hat{x}=(x, y)$. Boundedness is automatic, but continuity at the cross-section needs to be shown. 


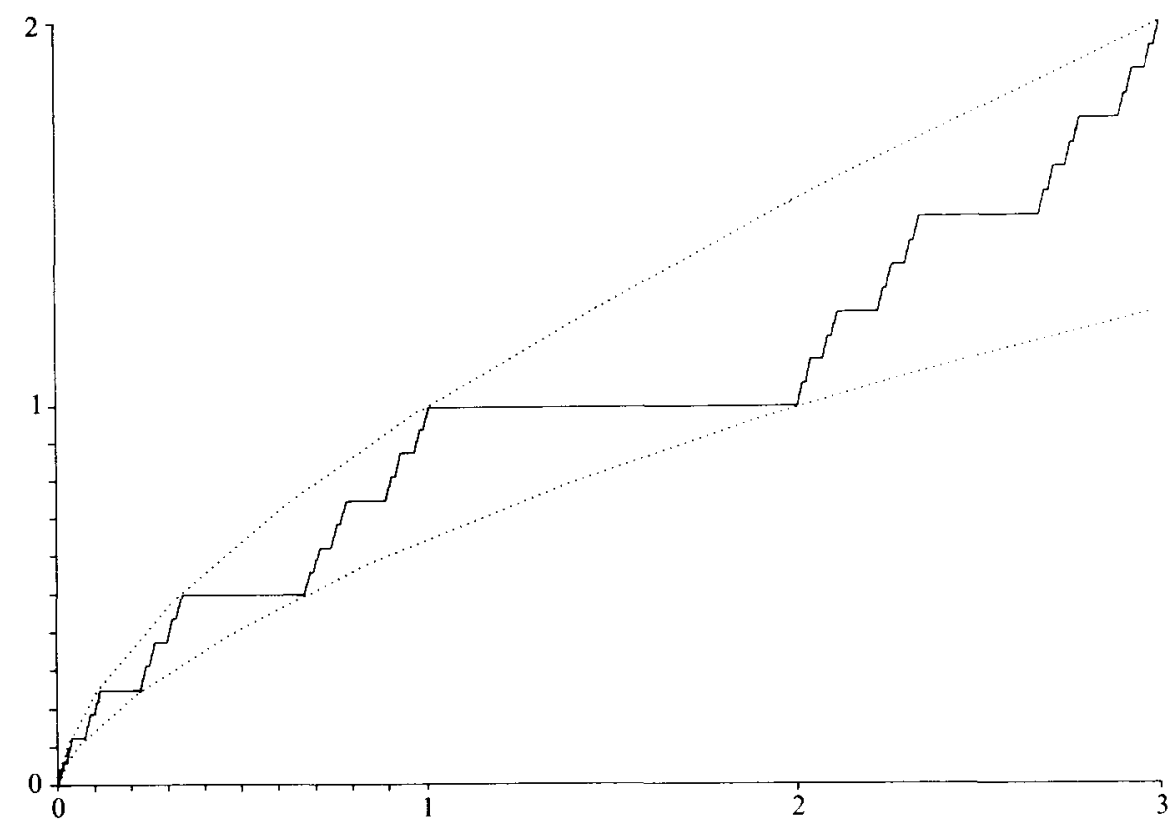

Figure 1. The extended Cantor function $L_{\hat{0}}(t)$.

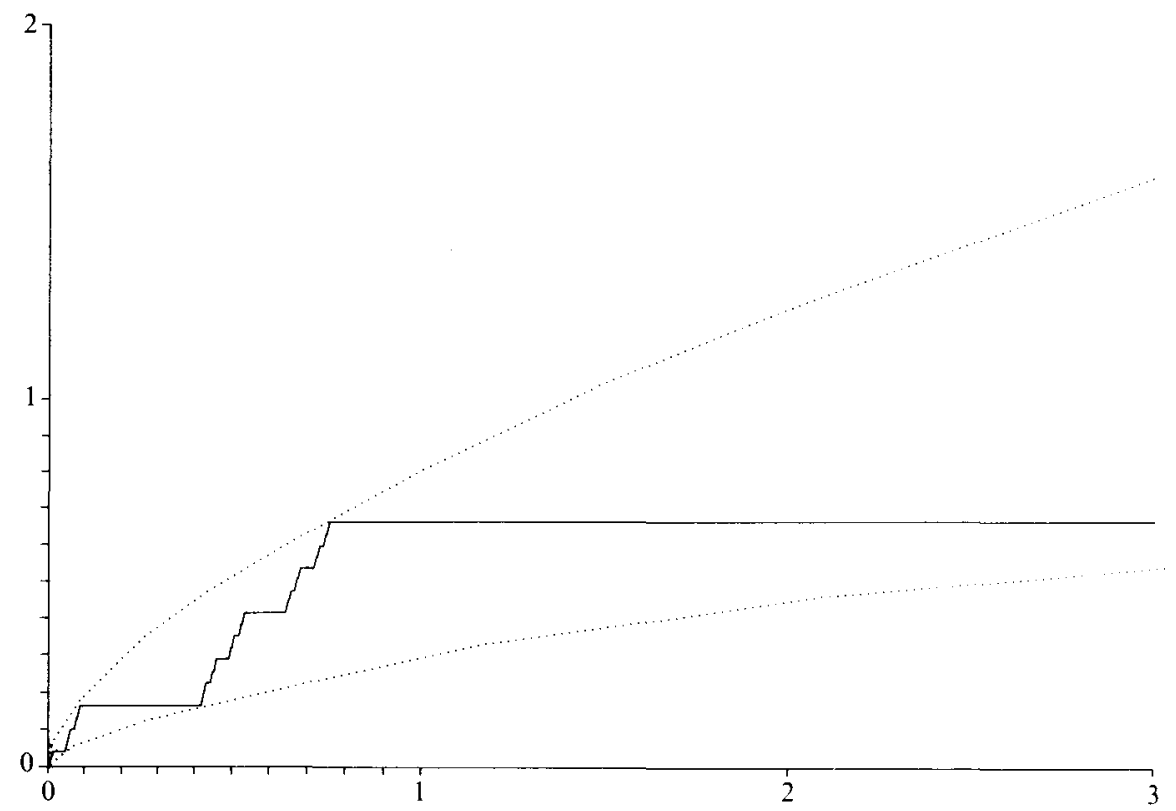

FIgure 2. The function $L_{\hat{x}}(t)$ for $\hat{x}=\left(\frac{1}{4}, \frac{3}{4}\right)$. 
First, for each $\hat{x}$ we define the function $f_{\hat{x}}: \mathbb{R} \rightarrow \mathbb{R}$ by $f_{\hat{x}}(t)=f(\hat{x}, t)$ for $t \in[0, \log 3)$, and $f_{\hat{x}}(t)=f\left(\hat{S}^{k}(\hat{x}), t\right)$ for $t \in[k \log 3,(k+1) \log 3)$. Note that via this map, the flow $\Phi_{t}$ on $Y$ corresponds to the left-shift flow $\sigma_{t}$ on functions from $\mathbb{R}$ to $\mathbb{R}$.

Next, define $L_{\hat{x}}(t)=t^{d} f_{\hat{x}}(-\log t)$ for $t>0$, and 0 for $t=0$, which by boundedness of $f$ is the limiting value. Now for $t \in[0,1]$, we claim that $L_{\hat{x}}(t)=\mu[x, x+t]$. This is because of the conformal transformation property of $d$-dimensional Hausdorff measure plus the fact that $S$ is measure-preserving on $(C, \mu)$ (see $\S 3$ of [Be-Fi]). Therefore $L_{\hat{x}}$ is continuous for $t \in[0,1]$ and in particular at the points $\frac{1}{3}, \frac{1}{9}, \ldots$ which correspond to the cross-sections of $Y$. Therefore it is continuous for all $t$, and so $f_{\hat{x}}$ is also continuous, hence in $C B(\mathbb{R})$, the space of continuous bounded functions.

Now write $\nu$ for the measure on $(\Omega, \mathscr{B})$ which is the image of $m$ on the special flow $Y$. Write $\Omega_{1} \subseteq \Omega$ for the image of $Y$ under the map $\hat{x} \mapsto L_{\hat{x}}$, and $C B_{1}$ for the image of the map $\hat{x} \mapsto f_{\hat{x}}$. Give $C B$ the topology $\mathscr{T}(C B)$ of uniform convergence on compacts.

The next proposition summarizes what has been done so far. The proof of this is now straightforward from the definitions.

Proposition 2.1. $\Omega_{1}$ is a compact $\tau_{\mathrm{s}}$-invariant subset of $\Omega$, and $C B_{1}$ is a compact $\sigma_{s}$-invariant subset of $C B$. The maps $\hat{x} \mapsto f_{\hat{x}}$ and $\hat{x} \mapsto L_{\hat{x}}$ are homeomorphisms from $Y$ onto $C B_{1}$ and $\Omega_{1}$, and are measure-theoretic flow isomorphisms.

Here is an alternative description of the scaling flow $\left(\Omega_{1}, \tau_{s}, \nu\right)$ : for $\hat{x}=(x, y)$, and $t \in[0,1], L_{\hat{x}}(t)$ is, as we have said, equal to $\mu[x, x+t]$. (In [Be-Fi] we called this the local time of $\mu$ ar $x$, in analogy with P. Lèvy's local time for Brownian zero

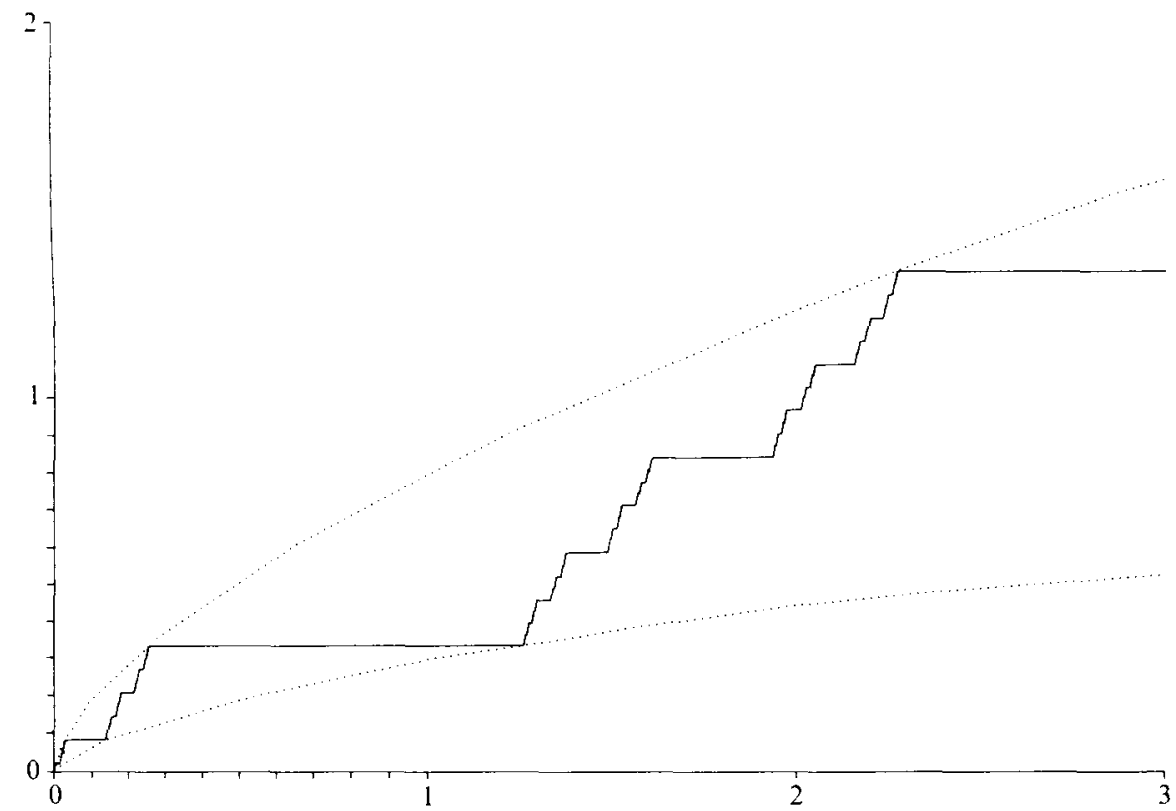

FIGURE 3. The function $L_{\hat{x}}(t)$ for $\hat{x}=\left(\frac{3}{4}, \frac{1}{4}\right)$. 
sets.) This gives a semiflow on the factor space of $\Omega$ which is the restriction to domain $[0,1]$. The path $L_{x}(t)$ is extended to $L_{\hat{x}}(t)$ successively on the intervals $[1,3],[3,9] \ldots,\left[3^{k}, 3^{k+1}\right]$ by choosing a preimage of $x$ in the $3 x(\bmod 1)$ map, taking its local time on $[1 / 3,1]$, and rescaling. These choices are indexed by the ternary expansion $y_{0}, y_{1}, \ldots$ of the point $y$ from the pair $\hat{x}=(x, y)$.

Examples. Let $\hat{x}=\hat{0} \equiv(0,0)=(x, y)$. Then $L_{\hat{0}}(t)$ is the usual Cantor function for $t \in[0,1]$, and for $t \geq 1$ is the extended Cantor function corresponding to the extended Cantor set $\hat{C}=\bigcup_{k=1}^{\infty} 3^{k} C$; this satisfies $L_{\hat{0}}(3 t / 2)=L_{\hat{0}}(t)$ for all $t$, i.e. $L_{\hat{0}}$ is a fixed point for $\tau_{\log 3}$. See Figure 1 for a graph of $L_{\hat{0}}$.

Other periodic examples correspond to $\hat{x}=\left(\frac{1}{4}, \frac{3}{4}\right)$ and $\hat{x}=\left(\frac{3}{4}, \frac{1}{4}\right)$, i.e. to the two periodic sequences $\hat{x}=(\ldots 202.020 \ldots)$ and its shift; see Figures 2 and 3 .

\section{Random integer Cantor sets}

Here we will define random versions of the integer Cantor set [ $C]$, and prove the order-two density exists for $[C]$, and for a.e. random integer Cantor set.

For an arbitrary non-decreasing function $L \in \Omega$, set $N(t)=[L(t)]$, where the brackets give the integer part. Now define a sequence $w$ for $k=0,1,2, \ldots$ by $w_{k}=N(k+1)-N(k)$.

Note that $N(0)=0$, that $|N(t)-L(t)| \leq 1$ for all $t$, and that the function $N(t)$ can be recovered from $w_{k}$ by the formulas:

$$
N(0)=0, N(n)=\sum_{k=0}^{n-1} w_{k}
$$

With a sequence $w^{+}=\left(w_{0}, w_{1}, \ldots\right) \in \Sigma^{+} \equiv \prod_{0}^{\infty}\{0,1\}$ we associate a subset $A$ of the non-negative integers $\mathbf{Z}^{+}$so that $\chi_{A}(k)=w_{k}$, i.e. $k \in A$ if and only if $w_{k}=1$.

Note, for example, that the integer Cantor set $[C] \equiv\left\{\sum_{i=0}^{M} x_{i} 3^{i}: x_{i}=0\right.$ or $\left.2, M \in \mathbb{N}\right\}$ is associated in this way with the extended Cantor function $L_{(0,0)}(t)$.

More generally for $\hat{x} \in \hat{C}=C \times C$, let $A_{\hat{x}}$ denote the subset of $\mathbf{Z}^{+}$corresponding to $L_{\hat{x}}(t)$. To define random integer Cantor sets we condition $x$ to be zero; this is equivalent to having $0 \in A_{\hat{x}}$. The measure $\mu$ on $C$ induces a probability measure on subsets of $\mathbf{Z}^{+}$via the map $y \mapsto A_{(0, y)}$ and therefore a measure on $\Sigma^{+}$, via $A \mapsto \chi_{A}$. We write $\tilde{\mu}$ for this measure; this defines the random version of $[C]$.

THEOREM 4.1. For $\mu$-a.e. $y \in C$, and for every rational $y$, setting $\hat{x}=(0, y)$, the order-two density of dimension $d$ of $A_{\hat{x}}$ exists, and is constant almost surely.

Proof. Since for any $\hat{x} \in \hat{C},\left|N_{\hat{x}}(t)-L_{\hat{x}}(t)\right| \leq 1$, we have that $\lim \left\|\tau_{s} N_{\hat{x}}-\tau_{s} L_{\hat{x}}\right\|_{[0,1]}^{\infty} \rightarrow 0$ as $s \rightarrow-\infty$, i.e. $N_{\hat{x}}$ and $L_{\hat{x}}$ are in the same unstable set in the scaling flow (are backwards asymptotic). Now by ergodicity of $\left(\Omega, \nu, \tau_{s}\right)$, one has for a.e. $\hat{x}$, by the Birkhoff ergodic theorem,

$$
\lim _{T \rightarrow \infty} \frac{1}{T} \int_{0}^{T}\left(\tau_{-s}\left(L_{\hat{x}}\right)\right)(1) d s=\int_{Y} f(\hat{x}, t) d \nu
$$


which is the expected value. By [Be-Fi] this is the one-sided order-two density, $c$, at $\mu$-a.e. point of $C$. For $y$ rational, one also has convergence since one is averaging a periodic function. Both limits pass over to $N_{\hat{x}}$ since the points $L_{\hat{x}}, N_{\hat{x}} \in \Omega$ are backward asymptotic.

THEOREM 4.2. For $\mu$-a.e. $y$, and for every rational $y$ which does not end in a sequence of all 2's, the dimension $d$ of $A_{\hat{x}}$ exists, and equals $\log 2 / \log 3$.

Proof. We wish to show that for $\hat{x}=(0, y)$, we have for every $\varepsilon>0$,

$$
n^{-\varepsilon} \leq N_{\hat{x}}(n) / n^{d} \leq n^{+\varepsilon} \quad \text { eventually. }
$$

Because $N_{\hat{x}}$ is backwards asymptotic with $L_{\hat{x}}$, it is equivalent to show

$$
t^{-\varepsilon} \leq L_{\hat{x}}(t) / t^{d} \leq t^{+\varepsilon}
$$

for $t$ large enough, or equivalently,

$$
e^{s e} \leq f_{\hat{x}}(s) \leq e^{-s e} \text { as } s \rightarrow-\infty .
$$

Now for $t \in\left[\frac{1}{3}, 1\right], L_{\hat{x}}\left(\frac{1}{3}\right) \leq L_{\hat{x}}(t) / t^{d} \leq 2 L_{\hat{x}}(1)$; so equivalently, for $t \in[0, \log 3]$,

$$
\frac{1}{2} f_{\hat{x}}(\log 3) \leq f_{\hat{x}}(t) \leq 2 f_{\hat{x}}(0) \text {. }
$$

Similarly, for $t \in[k, k+1]$,

$$
\frac{1}{2} f\left(S^{k+1} \hat{x}, 0\right) \leq f(\hat{x}, t) \leq 2 f\left(S^{k} \hat{x}\right) \quad \text { for all } k \in \mathbf{Z} .
$$

Now for all $\hat{x}, f(\hat{x}, 0) \leq 1$ which is certainly less than $e^{-s \varepsilon}$ as $s \rightarrow-\infty$, proving the upper bound. To prove the lower bound,

$$
\mu\left\{y: \frac{1}{2} f\left(S^{k+1} \hat{x}, 0\right)<e^{s \varepsilon} \text { infinitely often }\right\},
$$

for $k<K_{0}$, for $s \in[k, k+1]$, is zero by the Borel-Cantelli Lemma. The reason is that

$$
f\left(S^{k+1} \hat{x}, 0\right)<2^{(k+1) \varepsilon}
$$

when $S^{k+1}(\hat{x})$ is near the right endpoint of the Cantor set $C$, and this event has probability $2^{(k+1) \varepsilon}$. Since $\sum_{-\infty}^{0} 2^{(k+1) \varepsilon}<\infty$ for all $\varepsilon>0$, we are done. (The proof for rational $y$ is clear.)

The connection of integer with real fractal sets is also made through the following definition of the dimension of a measure at a point. This is based on Young, Ledrappier, and Misiurewicz [Y], [L-M]:

Definition. Let $\mu$ be a regular Borel measure on a metric space. Then the dimension of $\mu$ at $x$ is $\lim _{r \rightarrow 0} \log \mu\left(B_{r}(x)\right) / \log r$ if the limit exists, where $B_{r}(x)$ is the ball of radius $r$. On $\mathbb{R}$, we also define the one-sided dimensions, replacing e.g. $B$ by $B_{r}^{+}(x)=[x, x+r)$.

Proposition 4.3 For the measure $\mu$ on $C$, the symmetric and one-sided dimensions a.s. exist and equal $\log 2 / \log 3$. For every rational $y \in C$, the same is true for symmetric dimension, and for e.g. right-hand dimension if $y$ does not end in a string of 2's.

Proof. It is sufficient to consider the right-sided case, and to show that for every $\varepsilon>0, \varepsilon^{d+\varepsilon} \leq \mu[y, y+r] \leq \varepsilon^{d-\varepsilon}$ when $r$ is sufficiently small. The rest of the proof is just like that given above. 


\section{The tower and the odometer}

Now, as in the Introduction, let $a=\ldots a_{-1} . a_{0} a_{1} \ldots$ denote the sequence $\ldots 0000.101000101 \ldots$ in $\Sigma \equiv \prod_{-\infty}^{\infty}\{0,1\}$, where on the left we have all zeros, and on the right we have $\chi_{[C]}(n)$ for $[C]$ the integer Cantor set. (We use the decimal point to indicate the location of the zero coordinate in $\Sigma$.) Let $M$ be the orbit closure of $a$ in $\Sigma$, under the left shift $\sigma$.

First we will establish some basic facts about points $b \in M$. We write $0^{(0)}=0$, $1^{(0)}=1 ; 0^{(1)}=000,1^{(1)}=101 ; 0^{(2)}=0^{(1)} 0^{(1)} 0^{(1)}, 1^{(2)}=1^{(1)} 0^{(1)} 1^{(1)}=101000101$ and so on. We call $0^{(n)}, 1^{(n)}$ blocks of rank $n$. One sees that

(1) Every 1 is preceded and followed by a zero.

(2) No 1 is both preceded and followed by two zeroes.

(3) The same is true for blocks of rank $n$.

We will now show that these rules characterize points in $M$.

First, note that $\overline{0} \equiv \ldots 000 \ldots$ is in $M$. Next, assume that $b \in[1] \equiv\left\{b \in M: b_{0}=1\right\}$. We associate with $b$ a sequence $w=. w_{0} w_{1} w_{2} \ldots \in W \equiv \prod_{0}^{\infty}\{L, R\}$ according to the following construction:

By Rules 1 and 2 , we either see $000.101000=b_{-3} b_{-2} b_{-1} \cdot b_{0} b_{1} b_{2} b_{3} b_{4} b_{5}$, or $00010.1000=b_{-5} b_{-4} b_{-3} b_{-2} b_{-1}, b_{0} b_{1} b_{2} b_{3}$. If the decimal point occurs next to the left 1 , we write $L$ (as in the first case); if next to the right 1 , we write $R$.

One continues in this way for higher order blocks. It is clear that each sequence $w \in W$ determines a sequence in $\prod_{-\infty}^{\infty}\{0,1\}$ (indeed, at stage $n$ one has added at least $3^{n+1}$ symbols on each side). Furthermore since $a$ contains all the blocks $1^{(n)}$ (with the decimal place immedately to the left), each such $0-1$ sequence is in the orbit closure $M$, hence in [1] since $b_{0}=1$.

Let $\alpha$ denote this map from [1] to $W$. We have just shown that $\alpha$ is a bijection.

Next we define a map $\hat{\sigma}_{[1]}$ on [1]. This will be nearly the induced map $\sigma_{[1]}$ of $\sigma$ on [1], but is better behaved.

We know $b_{0}=1$. If $b_{1}, b_{2}, \ldots$ are all $=0$, then define $\hat{\sigma}_{[1]}(b)=a$. Otherwise, let $k$ be the least positive integer such that $b_{k}=1$, and define $\hat{\sigma}_{[1]}(b)=\sigma^{k}(b)$.

We will show that $\hat{\sigma}_{[1]}$ is isomorphic to the Kakutani-von Neumann dyadic odometer (also called the adding machine) $T$ on $W$. But first we recall the definition and some basic properties of $(W, T) . W=\prod_{0}^{\infty}\{L, R\}$ is a compact group (with the product topology) under coordinate-wise addition $L+L=L, R+L=R, R+R=L$ and carry $R$ to the right. $T(w)$ is defined by adding. $R L L L \ldots$ to $w$, so $T$ is a rotation of the group. With the binary representation $0 \sim L, 1 \sim R$ of $w$ as a point in the unit interval, $T$ has the graph (except for countably many points):

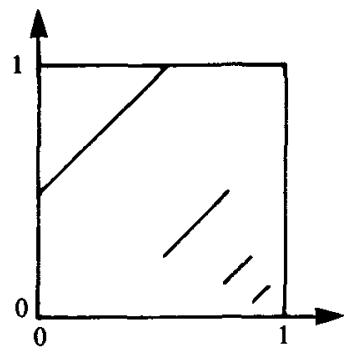


The transformation $(W, T)$ is uniquely ergodic, i.e. it has unique invariant probability measure $\eta$, which is Bernoulli $\left(\frac{1}{2}, \frac{1}{2}\right)$ measure on $\prod_{0}^{\infty}\{R, L\}$. There is a commutation relation of the (invertible) transformation $T$ with the non-invertible left shift $\sigma$ on $W$, which also preserves $\eta$ (but which is certainly not uniquely ergodic): for every $w \in W, T \sigma(w)=\sigma T^{2}(w)$. (This is easily verified directly, and one can also see it by drawing the graphs). The importance of this commutation relation becomes clear later. But first we have:

LEMMA 1. The map $\alpha:[1] \rightarrow W$ is a bijection which conjugates $\hat{\sigma}_{[1]}$ and $T$.

Proof. We have shown $\alpha$ is a bijection, and so now are to show that for every $b \in[1]$, $\alpha\left(\hat{\sigma}_{[1]}(b)\right)=T(\alpha(b))$. But this is easily checked by induction on the rank of the block containing the first 1 to the right of $b_{0}$, and by the observation that for the sequence $b$ corresponding to $w=. R R R .$. , the definition that $\hat{\sigma}_{[1]}(b)=a$ corresponds to the odometer turning over completely.

Next, given a (possibly infinite) invariant measure $\xi$ on $(M, \sigma)$, let $\xi_{1}$ denote the relative measure on [1], with the relative $\sigma$-algebra. We claim that $\xi$ is $\hat{\sigma}_{[1]}$-invariant. It is sufficient to verify this for cylinder sets, e.g. [.101] or [10.1], and one simply writes the inverse image as a (countable) disjoint union of cylinder sets, proving the claim. This leads to:

THEOREM 2. There is a unique non-atomic invariant measure $\rho$ on $(M, \sigma)$ such that $\rho([1])=1$.

Proof. Let $\rho$ on $M$ be non-atomic, invariant, with $\rho([1])=1$. The relative measure $\rho_{1}$ on [1] is an invariant probability measure; hence by Lemma 1 , and unique ergodicity of $T$, it must be Bernoulli $\left(\frac{1}{2}, \frac{1}{2}\right)$ measure under the correspondence $\alpha:[1] \rightarrow W$. But $\rho_{1}$ uniquely determines $\rho$, since given $\sigma$-invariance, if you know the measure of a cylinder set in $M$ with $b_{0}=1$ you know the measure of its translates, thus covering the case of all cylinders containing a 1 ; and the fixed point $\overline{0}$ has zero mass by non-atomicity. This proves uniqueness. Finally, the Bernoulli measure on [1] does give a $\sigma$-invariant measure on $M$, again by looking at cylinder sets.

We mention that drawing a picture of the tower can help one to visualize the cylinder-set argument, and that moreover such a picture is mathematically valid for the following reason: since the orbit of .LLL... has measure zero, and this is the only place where $\hat{\sigma}_{[1]}$ does not equal the induced map $\sigma_{[1]}$, the measure $\rho_{1}$ is also an invariant probability measure for $\sigma_{[1]}$.

Now we come to the last ingredient of our proof. Recall from $\S 4$ how $y \in C$ determines a random integer Cantor set $A_{\hat{x}} \subseteq Z^{+}$, where $\hat{x}=(0, y)$, with $1 \in A_{\hat{x}}$, and that $y$ maps to $z^{+} \in \Sigma^{+} \equiv \prod_{0}^{\infty}\{0,1\}$ where $Z^{+}=\left(z_{0}, z_{1}, \ldots\right)$ is the characteristic function of $\boldsymbol{A}_{\hat{x}}$. In this way Hausdorff measure $\mu$ determines a probability measure $\mu^{+}$on $\Sigma^{+}$. Let $\beta$ denote the map from $y$ to $z^{+}$. 
LEMMA 3. The following diagram commutes:
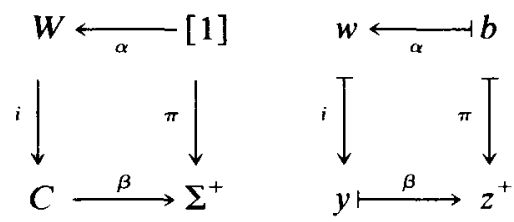

where $\pi\left(\ldots b_{-1}, b_{0} b_{1} \ldots\right)=\left(. b_{0} b_{1} b_{2} \ldots\right)$, and where the map $i$ from $w \in \Pi_{0}^{x}\{L, R\}$ to $y \in \Pi_{0}^{\infty}\{0,2\}$ is given by the substitution $L \rightarrow 0, R \rightarrow 2$. Furthermore $\pi$ is a bijection between [1] and the image $\beta(C)$. Also, $\pi$ is measure-preserving.

Proof. Note that $\alpha$ and $\beta$ are by definition measure-preserving. Also the map $i$ is, since it is just a relabeling of the $\left(\frac{1}{2}, \frac{1}{2}\right)$ Bernoulli shift. Furthermore $\alpha, i$ and $\beta$ are bijections, so once we verify the diagram commutes, we will be able to conclude that $\pi$ is a bijection and is measure-preserving.

Now it will be enough to show that for any fixed $n, w_{0} \ldots w_{n}$ and $y_{0} \ldots y_{n}$ determine the same initial sequence $z^{+} \in \Sigma^{+}$. One could write down a proof by induction but it is probably more useful to consider an example:

Say $y_{0}, y_{1}, y_{2}=2,2,0$, so $w_{0}, w_{1}, w_{2}=R, R, L$. Now $L_{(0, y)}(t)$ is determined for $t \in[0,27]$ and equals $\Delta_{1 / 27}\left(L_{r}(t)\right)$ where $r \in C$ has expansion $r=. y_{2} y_{1} y_{0} 000 \ldots=$ $.022000 \ldots$ Taking the integer part and the increments, we have the sequence

$$
z^{+}=. z_{0} z_{1} z_{2} \ldots z_{27}=.1000000000101000101000000000
$$

which is the same as the right half of that for . $R R L$.

Finally we are ready for the main theorem.

THEOREM 4. For the unique normalized non-atomic invariant measure $\rho$, for every $f \in L^{1}(M, p)$, we have for $\rho$-a.e. $x \in M$,

$$
\lim _{N \rightarrow \infty} \frac{1}{\log N} \sum_{k=1}^{N} \frac{S_{k}(f(x))}{k^{d}} \frac{1}{k}=c \int_{M} f(x) d \rho(x) .
$$

Proof. Since $\rho$ is ergodic (via Theorem 5.2), we know from Theorem 2.2 that it is sufficient to verify the theorem for one function in $L^{1}$. We take $f(x)=\chi_{[1]}(x)$. By ergodicity it is sufficient to check this for $x \in[1]$. But since, by Lemma 5.3, the relative measure $\rho_{1}$ agrees with that for the random Cantor sets (Theorem 4.1), we are done.

\section{Renormalization to a horocycle flow}

Now we will see how the transformation $(M, T)$ renormalizes to a flow on path space which also satisfies the order-two ergodic theorem. This is, by analogy to Fuchsian groups, a sort of 'horocycle flow', as explained in the notes at the end of the section.

Let $\hat{\Omega}$ denote $\{f: \mathbb{R} \rightarrow \mathbb{R}$ continuous with $f(0)=0\}$, the two-sided version of $\Omega$. Recall that any word $x$ in the base of the tower $(M, T)$ is uniquely defined on both sides by one half $\left(x_{0} x_{1} x_{2} \ldots\right)$. In exactly the same way, a path in $\Omega_{1} \subseteq \Omega$ extends uniquely to a path in a subset $\hat{\Omega}_{1} \subseteq \hat{\Omega}$. 
The scaling flow $\tau_{s}$ extends naturally to $\hat{\Omega}$; we write $\hat{v}$ for the corresponding $\tau_{s}$-invariant measure. Here is a second flow, which we call the increment flow (for reasons explained at the end of the section): define $\hat{\sigma}_{s}: \hat{\Omega} \rightarrow \hat{\Omega}$ by $\left(\hat{\sigma}_{s} f\right)(t)=$ $f(s+t)-f(s)$, for $s \in \mathbb{R}$. These satisfy the commutation relation $\hat{\sigma}_{b} \tau_{s}=\tau_{s} \hat{\sigma}_{e}{ }^{-s} b$; thus via the substitution $a=e^{s}$, one has an action of the $(a x+b)$ group.

We define horocycles for $\left(\hat{\Omega}, \tau_{s}\right)$ in the natural way, as the stable and unstable sets $W^{s}(f), W^{u}(f)$ of a point $f \in \hat{\Omega}$.

Let $\hat{\Omega}_{C}$ be that subset of $\hat{\Omega}_{1}$ corresponding to the base $\hat{C} \times\{0\}$ of the height-log 3 suspension flow of $\S 3$. Write $\hat{\Omega}_{H, 0}$ for the $\hat{\sigma}$-invariant set generated by $\hat{\Omega}_{C}$. Let $\hat{\Omega}_{H}$ be the $\tau$-invariant set generated by $\hat{\Omega}_{H, 0}$. Alternatively, $\hat{\Omega}_{H}$ is the $\hat{\sigma}$-invariant set generated by $\hat{\Omega}_{1}$, and also it is the joint $(\tau, \hat{\sigma})$-orbit closure of the extended Cantor function. We call the restriction of $\hat{\sigma}$ to $\hat{\Omega}_{H}$ the (negative) horocycle flow. One has the following picture: $\left(\hat{\Omega}_{H, 0}, \hat{\sigma}\right)$ is the height-one suspension flow over the tower $(M, \sigma)$. This is one ergodic component of the horocycle flow $\left(\hat{\Omega}_{H}, \hat{\sigma}\right)$. Here we give $\hat{\Omega}_{H}$ the natural $\hat{\sigma}$-invariant measure $\mu$. The other ergodic components are the continuum of slices $\hat{\Omega}_{H, a} \equiv \tau_{a}\left(\hat{\Omega}_{H, 0}\right)$ for $a \in[0, \log 3)$. Each component is uniquely ergodic in the sense that it has a unique normalized (infinite) non-atomic invariant measure. The flow $\tau_{s}$ permutes these components, via the commutation relation; however it does not preserve the measure $\mu$. For that correspondnence one needs a subflow of $\hat{\sigma}$ (by which we mean to move in an order-preserving way along a measured subset of the orbit):

For $f \in \hat{\Omega}$ any non-decreasing function, define the inverse of $f$ to be $f^{-1}(t)=$ $\sup \{s: f(s)=t\}$. On this subset $\hat{\Omega}^{+}$of $\hat{\Omega}$, define for $s \in \mathbb{R},\left(\tilde{\sigma}_{s} f\right)=\hat{\sigma}_{f_{(s)}^{-1}}(f)$.

LEMMA 1. $\tilde{\sigma}_{s}$ is a flow on $\hat{\Omega}^{+}$and satisfies $\tilde{\sigma}_{a} \tau_{s}=\tau_{s} \tilde{\sigma}_{e}^{-s d} a$.

Proof. It is easily checked that $\tilde{\sigma}_{s+t}=\tilde{\sigma}_{s} \circ \tilde{\sigma}_{t}$. Next, $\left(\tilde{\sigma}_{a} \tau_{s}\right)(f) \equiv \hat{\sigma}_{\left(\tau_{s} f\right)^{-1}(a)}\left(\tau_{s} f\right)=$ $\tau_{s} \hat{\sigma}_{e}^{-s}\left(\left(\tau_{s} f\right)^{-1}(a)\right)(f)=\left(\tau_{s} \hat{\sigma}_{f}^{-1}\left(e^{-s d} a\right)\right)(f) \equiv\left(\tau_{s} \tilde{\sigma}_{e}^{-s d} a\right)(f)$.

THEOREM 2. $\tilde{\sigma}_{s}$ is a measure-preserving flow on $(\hat{\Omega}, \hat{v})$. Its ergodic components are the sets $\hat{\Omega}_{a}$ corresponding to $\hat{C} \times\{a\}$, for $a \in[0, \log 3)$. For each $k \in \mathbf{Z},\left(\hat{\Omega}_{0}, \tilde{\sigma}_{3^{k}}\right)$ is isomorphic to the dyadic odometer. Each component flow is uniquely ergodic in the usual sense.

Proof. One sees first that $\tilde{\sigma}_{1}$ is the odometer, and for other $k$ it follows from the commutation relation, with $\tau_{k \log 3}$. The rest is clear from the construction.

We call $\left(\hat{\Omega}, \hat{\nu}, \tilde{\sigma}_{s}\right)$ the (negative) horocycle subflow.

Note that now the relationship between the one-sided shift $\sigma$ and the odometer $T$ of $\S 4, T \sigma=\sigma T^{2}$, corresponds exactly to:

$$
\tilde{\sigma}_{1} \tau_{-\log 3}=\tau_{-\log 3} \tilde{\sigma}_{2} \text {. }
$$

Furthermore, we remark that since $\tau$ and $\tilde{\sigma}$ preserve the same measure, the time-s and time- $t$ maps $\tilde{\sigma}_{s}$ and $\tilde{\sigma}_{t}$ are isomorphic via the conjugacy $\tau$; this corresponds to the fact that $T$ is isomorphic to $T^{k}$ for any $k$. Of course, both $T$ and $\tilde{\sigma}$ are zero entropy.

And we have, in summary the following.

THEOREM 3. The horocycle flow $\left(\hat{\Omega}_{H}, \hat{\sigma}, \mu\right)$ splits into a continuum of ergodic components $\hat{\Omega}_{H, a}$, permuted by the scaling flow $\tau_{s}$ for $s \in[0, \log 3)$. Each of these is uniquely 
ergodic in the sense that it has a unique non-atomic (infinite) invariant measure. The orbit of each $f \in \hat{\Omega}_{H}$ under $\hat{\sigma}$ is exactly the unstable set $W^{u}(f) \cap \hat{\Omega}_{H}$. Each component satisfies the order-two ergodic theorem.

Finally, we will describe the content of Theorems 2 and 3 pictorially. First, consider the ergodic component $\left(\hat{\Omega}_{H, 0}\right)$ of $h_{t}$. One can view $h_{t}$ as the suspension flow of height one over the tower transformation $(M, \rho, \sigma)$, and so $\hat{\Omega}_{H, 0}=M \times[0,1]$ modulo identification of the top and bottom of the flow. See Figure 4.

Note that the tower heights are, indeed, coded from the odometer by the formula: (return time to base) $=3^{k}+1$ where $\omega_{k}$ is the first occurrence of the symbol $L$ in $\omega=\left(\omega_{0}, \omega_{1}, \ldots\right) \in W$. Next let $\bar{h}_{t}$ denote the induced flow of $h_{t}$ on the subset $W \times[0,1]$ of $\Omega_{H, 0}$. This flow is exactly the suspension flow of the odometer, and preserves Lebesgue $\times$ Bernoulli measure. An $\tilde{h}_{t}$ is a subflow of $\bar{h}_{t}$, which is also like a suspension flow of the odometer, except that one now jumps along a Cantor set of times. See Figure 5.

Note that $\tilde{h}_{t}$ and $\vec{h}_{t}$ are naturally isomorphic, via the singular time change given by the Cantor function: $C \rightarrow[0,1]$. Now the scaling flow $\tau_{s}$ is a height $\log 3$ suspension flow over $(C \times C, S)$, which we draw horizontally. Vertical slices give the ergodic components of $\tilde{h}_{t}$. See Figure 6.

However we see from the relation $\hat{\sigma}_{e^{a}} \tau_{a}=\tau_{a} \hat{\sigma}_{1}$ that the levels in the slice $\hat{\Omega}_{H, a}$ are separated by distance $e^{a}$. So the entire picture looks like this, where the arrowed rectangle denotes the commutation relation of $\tau_{s}$ and $h_{t}$. Note that $\tau_{s}$ acts on the whole tower, but compresses the infinite measure toward its finite invariant measure. See Figure 7.

Concluding remarks. In summary, one can now see clearly the role of the commutation relation $T \sigma=\sigma T^{2}$. Fractal sets are created from an interplay of two structures: scaling and translation; and here the map $T$ corresponds to translation, while $\sigma$ corresponds to geometrical scaling, for the integer Cantor sets. As we have just seen, the pair $(\sigma, T)$ can be renormalized to a pair of flows on $\Omega$, which preserve the commutation relation, and which reflect the geometry of the middle-third Cantor set. This renormalization takes place not just as a weak convergence of measures on path space, but also in a stronger almost-sure form. Almost every point in the

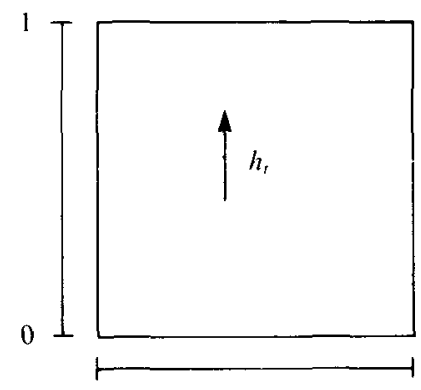

$(M, \sigma)$

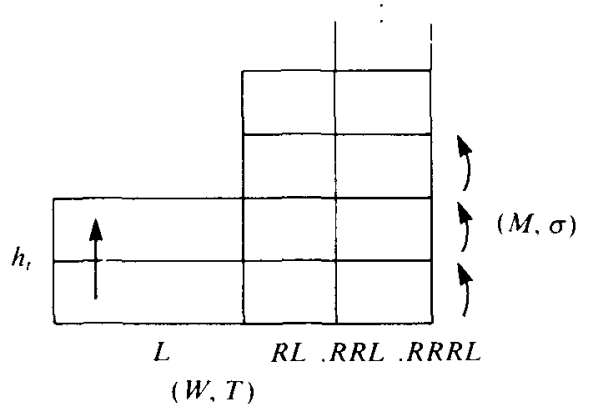

$(W, T)$ 

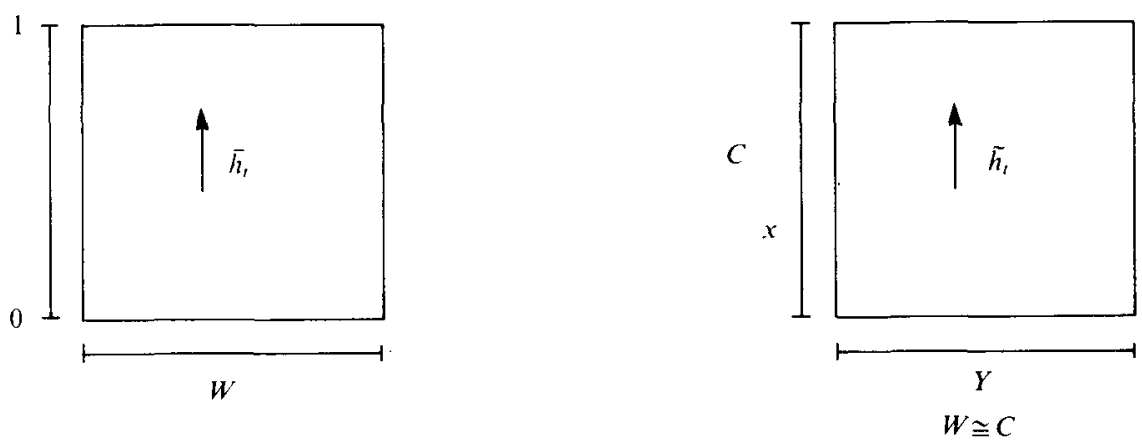

FIGURE 5

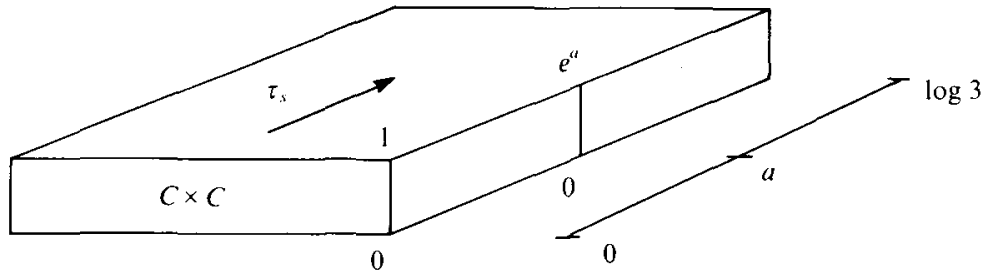

Figure 6

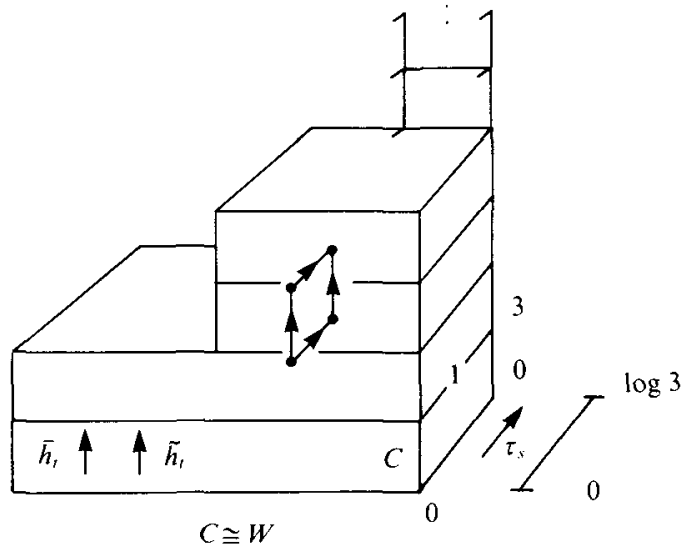

Figure 7

tower $(M, \sigma)$, when viewed as an element of $\hat{\Omega}$, is a generic point under scaling for $\left(\hat{\Omega}_{1}, \nu, \tau_{-s}\right)$; this follows from a well-known theorem of Kriloff and Bogoliouboff, i.e. from the Birkhoff ergodic theorem plus compactness. Then the horocycle flow can be recovered from this by shifting via $\hat{\sigma}$; and so a.e. $0-1$ sequence in the tower contains all the information for both flows, and renormalizes in this sense to the continuous model.

The ideas which led to this paper came from two quite different sources: from probability theory, and from the geometry of Fuchsian groups. This was written in 
part to serve as an introduction to those examples, which will be studied separately in later papers. Here is a sketch of the various connections.

The unifying structures are: a joint action of two flows which satisfy the commutation relation, the renormalization of a discrete model to a continuous one, and a dichotomy between the cases of flnite and infinite measures.

First we look at the probability examples. Let $\nu$ be a $\tau$-invariant measure (for some dimension $d$ ) on a path space $\Omega$, which has been given a Polish topology. Then invariance of $\nu$ corresponds exactly to what is known as a self-similar process; see [T] for a wealth of references. What we have called the increment flow $\hat{\sigma}$ also plays a role in this theory (see [V]): the process has stationary increments if and only if $\nu$ is $\hat{\sigma}$-invariant.

We studied the scaling flow (with $d=\frac{1}{2}$ ) for two examples, Brownian motion and Lévy's local time, in [Fi 1,2] and [Be-Fi] respectively. In both settings one has the generic point statement and a.s. renormalization of discrete-time processes, e.g. the simple random walk $S_{n}$ for $\nu=$ Wiener measure, and the number $N_{n}$ of zeroes of $S_{n}$ for local time. The increment flow is also interesting for these examples.

First, for Brownian paths, $\hat{\sigma}_{s}$ corresponds to the left shift flow on the derivatives of the paths, i.e. on white noise. This gives a model for white noise as a Bernoulli flow (of infinite entropy) on a Lebesgue space.

Restricted to the polygonal paths of $S_{n} \in \Omega$, the time-one map $\hat{\sigma}_{1}$ corresponds to left shift on the increments $X_{i}$. Since $S_{n}$ is a generic point for the scaling flow, in this sense the Bernoulli shift $X_{i}$ renormalizes to white noise. The various time-t maps of $\hat{\sigma}$ are conjugated by the scaling flow, and this agrees with the fact from Ornstein theory that all infinite entropy Bernoulli transformations are isomorphic (compare Theorem 2 above). Note that for this example, $\hat{\sigma}$ and $\tau$ both preserve the same (probability) measure $\nu$.

For local time, one has a situation closer to the Cantor set case: $\hat{\sigma}$ now preserves an infinite measure, but the subflow $\tilde{\sigma}$ (defined exactly as for the Cantor set) preserves $\nu$. Again, one can prove an order-two ergodic theorem; the key step (order-two density of random walk zeroes) is done in [Be-Fi]. We mention that these two examples - the Cantor set scaling flow and the Brownian local time process - again can be thought of as self-similar processes with stationary increments. But to do this one must now either use the infinite measures, or look instead at the corresponding (very discontinuous) processes given by the inverse paths.

In both the Brownian and local time cases, one can show (by a Borel-Cantelli argument) that the orbits of $\hat{\sigma}$ a.s. remain within the stable set of a path. So one can think of the increment flow as a horocycle flow, because of the geometrical analogy.

That such an analogy might exist at all could be suggested by the similarity of the commutation relations. Dan Rudolph made this key observation when we were discussing the local time and the integer Cantor set examples, and following up on his idea, one sees that this apparent connection is, indeed, no accident at all.

The classical situation is exemplified by Fuchsian groups of first type; see e.g. $[B o, M]$ for background. In that case the geodesic and horocycle flows preserve the same (finite) measure, and satisfy the commutation relation. For groups of second 
type, however, the horocycle flow would have to jump along a perfect set of times. But as in the Cantor set case, this is not so intractible: instead one should start with the full horocycle flow $h_{t}$, for which the natural invariant measure is infinite; its subflow $\tilde{h}$ is the object which commutes nicely with the geodesic flow. This is uniquely ergodic and zero entropy, much like the odometer. And again, one can prove an order-two ergodic theorem for $h_{t}$, which is, by interchanging 0 and $\infty$, dual to proving the existence of order-two density for the Patterson measure on the limit set. Full details will be given in a later paper; a third paper will treat Kleinian limit sets with a similar approach.

Postscripts. As Jon Aaronson pointed out to us, the tower can also be built in this way: take the triadic adding machine on $\Pi_{0}^{\infty}\{\overline{0}, \overline{1}, \overline{2}\}$, and now label the measure zero subset $\Pi_{0}^{\infty}\{\overline{0}, \overline{2}\}$ with the symbol 1 and its complement with 0 . Thus our tower is the tower of an invariant measure-zero subset of the triadic adding machine over the dyadic adding machine. This leads to a natural generalization of the present example: take a $k$-adic odometer sitting inside of an $\boldsymbol{m}$-adic odometer. Again one has a nice correspondence between real and integer Cantor sets, and one can prove (by the same method), an order-two ergodic theorem. For some related constructions see [Aa 6].

Aaronson has shown us how, using a cutting and stacking construction of the tower, one can find a recurrence formula for $a(n) \equiv \int_{A} a(n, x) d \rho$ where $A$ is the base [1] of the tower. This leads to an expression for $c$ as the sum of an infinite series. T. Kamae has also (independently of Aaronson) found a method for approximating $c$ arbitrarily well.

Note added before publication: We have received a new preprint in which $\mathrm{N}$. Patzchke and M. Zähle also give the approximate value for $c$.

\section{REFERENCES}

[Aa 1] J. Aaronson. Rational ergodicity and a metric invariant for Markov shifts. Israel J. M. 27 (1977), 93-123.

[Aa 2] J. Aaronson. On the ergodic theory of non-integrable functions and infinite measure spaces. Israel J. M. 27 (1977), 163-173.

[Aa 3] J. Aaronson. On the pointwise behaviour of transformations preserving infinite measures. Israel J. M. 32 (1979), 67-82.

[Aa 4] J. Aaronson. The asymptotic distributional behaviour of transformations preserving infinite measures. J. D'Anal. Math. 39 (1981), 203-234.

[Aa 5] J. Aaronson. The intrinsic normalizing constants of transformations preserving infinite measures. J. D'Anal. Math. 49 (1987), 239-270.

[Aa 6] J. Aaronson. Rational ergodicity, bounded rational ergodicity and some continuous measures on the circle. Israel J. M. 33 (1979), 181-197.

[Aa-De 1] J. Aaronson \& M. Denker. Upper bounds for ergodic sums of infinite measures preserving transformations. Trans. Amer. Math. Soc. 319 (1990), 101-138.

[Aa-De 2] J. Aaronson \& M. Denker. Lower bounds for partial sums of certain positive stationary processes. In: Almost Everywhere Convergence. Proceedings. G. A. Edgar and L. Sucheston, eds. Academic, New York, 1989, pp 1-9.

[Aa-De-Fi] J. Aaronson, M. Denker \& A. M. Fisher. Second order ergodic theorems for ergodic transformations of infinite measure spaces. Proc. Amer. Math. Soc. 144 No. 1 (1992), 115-128.

[Aa-S] J. Aaronson \& D. Sullivan. Rational ergodicity of geodesic flows. Ergod. Th. \& Dynam. Sys. 4 (1984), 165-178. 
[Be-Fi] T. Bedford \& A. M. Fisher. Analogues of the Lebesgue density theorem for fractal subsets of the reals and integers. Proc. London Math. Soc. 64 (1992), 95-124.

[Bo] R. Bowen. On axiom $A$ diffeomorphisms. Conf. Board Math. Sci. 35 (1978), American Math. Soc., Providence RI, 1978.

[Fa] K. J. Falconer. The geometry of fractal sets. Cambridge University Press, New York/ Cambridge, 1985.

[Fi 1] A. M. Fisher. Convex-invariant means and a pathwise Central Limit Theorem. Adv. Math. 63 (1987), 213-246.

[Fi 2] A. M. Fisher. A pathwise Central Limit Theorem for random walks. Ann. Prob. to appear.

[Fi 3] A. M. Fisher. Analogues of geodesic and horocycle flows for certain fractal sets. In preparation.

[Ha-I-K] A. Hajian, Y. Ito \& S. Kakutani. S. Kakutani: Selected Papers. Vol. 2. Birkhäuser, BostonBasel, 1986

[Ho] E. Hopf. Ergodentheorie. Chelsea, New York, 1948.

[L-M] F. Ledrappier \& M. Misiurewicz. Dimension of invariant measures for maps with exponent zero. Ergod. Th. \& Dynam. Sys. 5 (1985), 595-610.

[M] B. Marcus. Ergodic properties of horocycle flows for surfaces of negative curvature. Ann. Math. 105 (1977), 81-105.

[T] M. S. Taqqu. A biliographical guide to self-similar processes and long-range dependence. In: Dependence in Probability and Statistics. E. Eberlein and M. S. Taqqu, eds. Birkhäuser, Boston-Basel, 1986, pp. 137-162.

[V] W. V. Vervaat. Sample path properties of self-similar processes with stationary increments. Ann. Prob. 13 (1985), 1-27.

[W] D. V. Widder. An Introduction to Transform Theory. Academic Press, New York-London, 1971.

[Y] L. S. Young. Dimension, entropy and Lyapunov exponents. Ergod. Th. \& Dynam. Sys. 2 (1982), $109-124$. 